\title{
QualiTrack: Highspeed TUI Tracking for Tabletop Applications
}

\author{
Ramon Hofer, Thomas Nescher, and Andreas Kunz \\ ETH Zurich, inspire, Tannenstrasse 3, \\ 8092 Zurich, Switzerland \\ \{hofer, nescher\}@inspire.ethz.ch, kunz@iwf.mavt.ethz.ch
}

\begin{abstract}
In this paper, we present a new technology to track multiple active Tangible User Interfaces (TUI) on a projection table. We use a commercial high speed infrared tracking camera with modified firmware. With a special tracking method, we reach update rates of up to $250 \mathrm{~Hz}$ with low latencies. At this tracking rate, we are able to track the position, state and the orientation of more than 10 active TUIs on the table. For this, we use specified bit codes which are transmitted by the devices. We developed dedicated hardware (SyncUnit) and software to keep the devices and the high speed camera synchronized. The system of camera, SyncUnit, and devices is fully hardware controlled and delivers event coded tracking data for further usage in interactive applications.
\end{abstract}

Keywords: Single Display Groupware, Tangible User Interfaces, CSCW, Tracking, high speed, low latency.

\section{Motivation and Related Work}

In applications such as brainstorming, design review and general idea finding tasks, interfaces are needed that satisfy the need for highly responsive and intuitive interactions. Sketches and fast drawing or writing gestures are used to exchange information between collaboration partners. Thus, it is essential that multiple strokes - being sketched very fast by different users on the same screen - are reliably tracked and visualized in order to provide a real and intuitive feeling to the users. In addition, hiding technology but still providing all advantages is a key issue in this special field of human computer interaction. In this paper, a technical approach is shown to support such tasks by providing a high speed multi device tracking system.

Many researchers have addressed tracking of multiple objects on a screen. One of the most popular methods for multi-device tracking on horizontal surfaces is optical tracking, where a camera is placed above or behind a projection screen representing the interactive surface. The TUIs are either identified by shape recognition as in the DigitalDesk setup [1], by marker detection as in the InfoTable [2], by occlusion detection as in [3], or by color detection [4]. Using marker or color tracking provides the possibility to unambiguously track several devices at once, since each device can contain a unique optical identification pattern (fiducials). When using infrared light (IR), the tracking is no longer visible to the user. 
Simulated table top tennis [5] is one of the very responsive systems that provides update rates of $60 \mathrm{~Hz}$ and latencies of only $50 \mathrm{~ms}$ by using commercial ART cameras. In [6], an infrared tracking system was developed which is able to track position, orientation and state of up to 7 interactive devices on a table. The final update rate was $20 \mathrm{~Hz}$. By using device specific bit codes, simultaneous identification of all active devices was possible, but the tracking rate was low.

Up to now, there is no system which provides a high update rate $(>100 \mathrm{~Hz})$ with reliable identification of multiple devices and determination of their states as well.

\section{System Components}

The QualiTrack system is an optical system using a high speed IR camera and a back projection onto an opaque table. Each interactive device has an integrated IR receiver, at least one IR LED, and also a signal processor. The devices detect an IR synchronization signal with their receivers and answer with a unique device code (a binary code of $\mathrm{n}$ bits). This code is flashed back bit per bit (IR LED on/off) upon every synchronization signal. At every synchronization signal, a camera frame is recorded and processed. When as many frames as the bit code length are triggered and recorded, the recorded camera frames are combined and the code, the position, as well as the orientation of the devices are determined. A dedicated hardware device, the SyncUnit, was developed, which handles the synchronization of all components, i.e. IR flash, camera trigger and data transfer from the camera to the PC host system.

A commercial high speed motion tracking camera from Qualisys, the MCU1000 (up to $1000 \mathrm{~Hz}$ ) is used. In order to achieve high resolution at a high update rate, the camera is used at $250 \mathrm{~Hz}$, at which it still provides the full resolution of $658 \mathrm{x} 496$ ( $>250 \mathrm{~Hz}$, the field of view is reduced). The camera contains an efficient hardwarebased blob detection and directly delivers the coordinates of detected IR points.

A dedicated SyncUnit (controlled by an ARM7 board) ensures that the camera and the interaction devices run in sync all the time. This synchronization is independent of the PC host system and runs on its own. The SyncUnit generates the sync IR flash to trigger the devices and it also triggers the camera. After that, the preprocessed data from the camera is received. USB is used to transfer the data from the SyncUnit to the
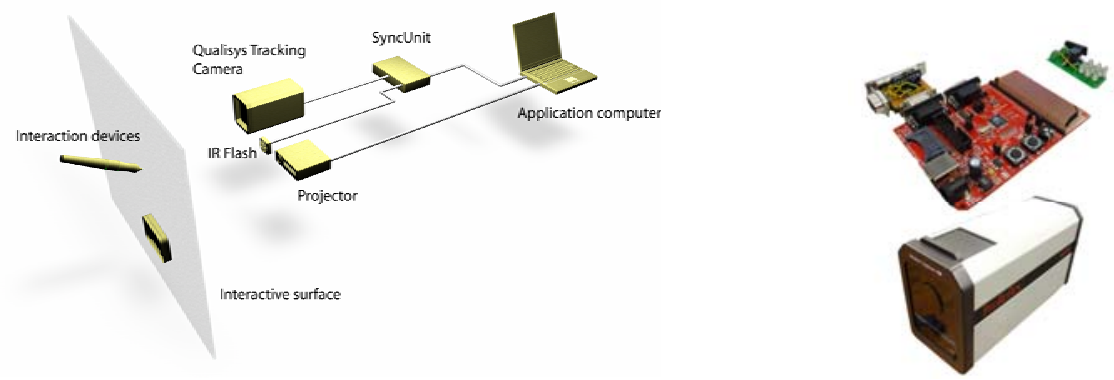

Fig. 1. Left: The QualiTrack setup. Right top: SyncUnit (ARM7 Board) and the IR Syncflash LED array. Right bottom: The Qualisys Camera ProReflex MCU1000 [7]. 
PC. A Java application processes the LED position data into TUI data. This includes the computation of TUI center points, their position, rotation, acceleration, rotational acceleration, and path prediction. The processed data is then sent to a client application via network using the TUIO [8] protocol.

The system distinguishes between two different kinds of devices: Devices with only one IR LED for drawing and pointing, and devices with 3 IR LEDs. The latter can also be tracked in orientation for other interaction possibilities. By pressing the devices onto the screen, a switch can be activated to change the state. An example for such a device is the Handle Tool. It serves as a navigation device. By pressing and rotating it the drawing area is zoomed. Shifting and pressing will drag the drawing.

\section{System Characteristics}

In order to unequivocally identify the TUIs, several post processing steps are required on the PC host system. Per frame, the SyncUnit transmits a set of LED position coordinates and the bit position number to the PC host. As every device has its unique nbit code, the software on the PC host has to analyze $n$ camera frames to identify the device. Hence, the real output frequency of the system of identified TUIs would drop to the camera frame rate divided by $n$. The smaller $n$ is, the faster the system is, but the less TUIs can be used in parallel. To solve this problem, the devices' movements are predicted and each device's position is updated every time the camera sees it. This means that whenever a device enters the camera's field of view, the system has to wait for $\mathrm{n}$ frames to identify it (initialization phase). But for every subsequent frame, the device's position is updated whenever its bit code is 1 for a frame (LED switched on). Thus, the number of subsequent 0 in a device's bit code limits the update rate.

An 8-bit code was chosen for the system, in which only a single 0-bit is allowed between two 1-bits. Hence, the update rate only drops to half the camera frame rate. In order to further improve the tracking quality, the device's position is extrapolated using its previous velocity and acceleration. Thus, the device's position can be output at every camera frame using extrapolation. Although not all combinations of an 8-bit code can be used anymore, there is still a sufficient number of available IDs for all devices on the interactive surface of the table. Actually, this coding scheme would allow for having devices with low update rate as well (i.e. many subsequent 0 ).

There is another problem if a bitcode has many 1-bits: If many devices are used in parallel, they can also be active in the same camera frame. Thus, more potential collisions (extrapolated LEDs lighting up at the same location than another device's LED) are likely in particular if devices are close to each other. By employing position extrapolation, the area in which two devices could collide can be narrowed. Additionally, the bitcode should be chosen so that few devices have a 1-bit at the same bit position in order to minimize collisions. Apart from the 8-bit code, 2 more arbitrarily usable bits are appended to the code, which allow each device to signal its status to the PC host. This is used for instance to check whether a button is pressed. Thus, a status update is only possible after every 10th frame. The device codes were set with respect to the aspects mentioned before: update rate and collision prevention (see Fig. 2). For proofing the system's responsiveness, a pong game was realized. 


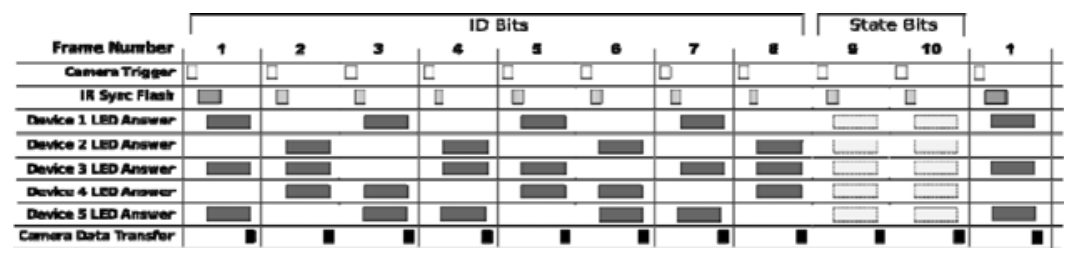

Fig. 2. Bit codes of the first 5 devices and timing of all components ( 1 Frame $=4 \mathrm{~ms}$ )

\section{Conclusion and Future Work}

A high speed tracking system for the detection of tangible user interfaces on a back projection tabletop surface is presented. It provides update rates of $250 \mathrm{~Hz}$ for fast interactions of up to $1 \mathrm{~m} / \mathrm{s}$ with reliable object identification and state recognition. The system forwards tracking data using the TUIO protocol to any TUIO compatible client. The amount of interactive devices is not restricted, but compromises in update rate and collision avoidance have to be made. We are currently working on creativity applications that need high spatial and temporal accuracy and low latencies, such as writing and sketching applications. It is planned to evaluate if such high update rates are necessary at all for the envisioned interactions. The goal is to set up a taxonomy that shows which low update rates are no longer tolerable for such applications as well as thresholds, above which high update rates are unnecessary.

\section{References}

1. Wellner, P.: Interacting with Paper on the DigitalDesk. Communications of the ACM 36(7) (1993)

2. Rekimoto, J., Saitoh, M.: Augmented Surfaces: A Spatially Continuous Work Space for Hybrid Computing Environments. In: Proceedings of the SIGCHI conference on Human factors in computing systems: the $\mathrm{CHI}$ is the limit, David Lawrence Convention Center Pittsburgh, Pennsylvania, USA, ACM Press, New York (1999)

3. Smart Technologies, DViT (Digital Vision Touch) Technology, http: / / www2 . smarttech.com/st/de-DE/Products / SMART+Boards / Overlays/

4. Nishimoto, K., Amano, K., Usuki, M.: pHotOluck: A Home-use Table-ware to Vitalize Communication at Mealtimes by Projecting Photos onto Dishes. In: TableTop, Adelaide, Australia. ACM Press, Adelaide (2006)

5. Rusdorf, S., Brunnett, G.: Real Time Tracking of High Speed Movements in the Context of a Table Tennis Application. In: VRST 2005, ACM Press, Monterey (2005)

6. Ganser, C., Steinemann, A., Kunz, A.: InfrActables: Multi-User Tracking System for Interactive Surfaces. In: IEEE VR 2006, Alexandria/Virginia, USA (2006)

7. Qualisys Motion Tracking Systems, http://www. qualisys.com/

8. Kaltenbrunner, M.: TUIO - A Protocol for Table Based Tangible User Interfaces. In: GW 2005, Vannes, France (2005) 